\title{
Smartphone-based arrhythmia detection: Should we encourage patients to use the ECG in their pocket?
}

\author{
Ryan D. White and Greg Flaker* \\ University of Missouri, Department of Medicine, Division of Cardiovascular Diseases, Columbia, USA
}

\section{Introduction}

As of 2015, 64\% of American adults owned a smartphone, up from $35 \%$ in the spring of 2011[1]. These devices have become ingrained in our lives, and their utility is ever expanding. Once used for merely communication, smartphones have come to replace the wristwatch, provide camera and navigation functions, and allow easy access to the Internet. More recently, they have become powerful tools in monitoring our health. There are smartphone-enabled glucometers, blood pressure cuffs, oximeters, and even heart monitors. This will present a new challenge to physicians, namely the interpretation of diagnostic information captured on smartphones, in particular cardiac arrhythmias. Atrial fibrillation (AF) is a common arrhythmia, affecting more than 2.7 million Americans [2]. This arrhythmia is associated with significant morbidity, carrying a 4 - to 5 -fold increased risk for ischemic stroke [3]. AF is often silent, with patients occasionally presenting with stroke as the first manifestation of the arrhythmia [4]. Other patients have troubling symptoms such as palpitations or dizziness, but traditional monitoring has been unable to define an arrhythmia. Periodic sampling of heart rate and rhythm could be helpful to establish a diagnosis in these conditions. Smartphone monitoring of AF could also prove useful in patients with known AF. Symptomatic episodes could be documented which might alter the patient's regimen of rhythm control or rate control medications. Furthermore, the costs for treating AF are extremely high, accounting for greater than 6.5 billion dollars annually [5]. An investigation into the use of relatively inexpensive smartphones as a monitoring device for $\mathrm{AF}$ is attractive.

The purpose of this manuscript is to investigate the current landscape of smartphone-based arrhythmia detection and monitoring. We will discuss the current technologies, and the methods used to validate them. It is our hope to provide practicing clinicians with the background information necessary to critique smartphone-based event monitoring.

\section{Smartphone technology}

Currently, the methods of using a smartphone to detect and monitor atrial fibrillation can be divided into two groups. The first group simply uses a downloadable application and hardware that already exists on modern smartphones, the camera and lamp. The second group uses a pair of external electrodes, either built into the case or as a standalone unit that communicates with an application downloaded to the phone. The requirement of purchasing additional hardware is a possible barrier to use. As discussed, methods of using a smartphone to screen for atrial fibrillation are being developed that use a downloadable application, without the need of additional hardware. These applications use the phone's camera and lamp, in effect turning the phone into a photoplethysmographic (PPG) sensor (Figure 1). The

phone's lamp illuminates the user's finger, and a signal recorded through the phone's camera is then processed through an algorithm. The algorithm analyzes the regularity of the pulse waves. One such standalone smartphone PPG application currently in development is Cardio Rhythm, developed by Cardio (Cambridge, MA, USA, Figure 1). The accuracy of this application to detect atrial fibrillation was shown in an outpatient clinic in 1013 patients with known hypertension, diabetes mellitus, and/or aged $>65$ years [6]. Immediately following completion of a single-lead ECG (using the AliveCor device discussed below), 3 PPG waveforms were acquired sequentially from each patient using an iPhone 4S (Apple Inc) running the Cardio Rhythm smartphone application (Cardio Inc). Each PPG waveform recording lasted 17.1 seconds and was classified automatically by the Cardio Rhythm smartphone application as "Regular" or "Irregular." A diagnosis of AF was produced if at least 2 of 3 PPG waveform recordings from a single patient were classified as "Irregular." The approach for detecting the presence of AF was based on a lack of repeating patterns in the PPG waveform because of the irregular rhythm of AF. When a diagnosis of AF was made by the Cardio Rhythm application, the AliveCor automated AF detection algorithm, or both, a full 12-lead ECG was performed within 15 minutes of the initial screening. Two blinded cardiologists over-read the single-lead ECG printouts to provide a reference diagnosis by using standard criteria. They found that the diagnostic sensitivity and specificity of Cardio Rhythm for AF detection was $92.9 \%$ (95\% CI 77-99\%) and 97.7\% (95\% CI 97-99\%) respectively, suggesting that the application provides an accurate and reliable means to detect AF in patients at risk of developing AF. A disadvantage of the dependence on regularity of rhythm to define AF is that PVC's or PAC's may cause irregular rhythm.

One of the more recent smartphone-enabled health devices include smartphone-based event monitors which combine external ECG sensors with a smartphone application. One such device is the AliveCor Heart Monitor, a smartphone-based heart monitor that is capable of recording a single-lead ECG. This device received US FDA approval in 2012. The AliveCor Heart Monitor is smaller than a credit card, and consists of two metal electrodes. A bipolar lead I is created when the two metal electrodes are touched by the patient's right and left hands. The ECG electrical signals are then converted to an ultrasonic FM

Correspondence to: Greg Flaker, University of Missouri, Department of Medicine, Division of Cardiovascular Diseases, Columbia, USA, Tel: 573882 2296; Fax: 573884 3221; E-mail: FlakerG@health.missouri.edu

Received: March 21, 2017; Accepted: April 21, 2017; Published: April 24, 2017 

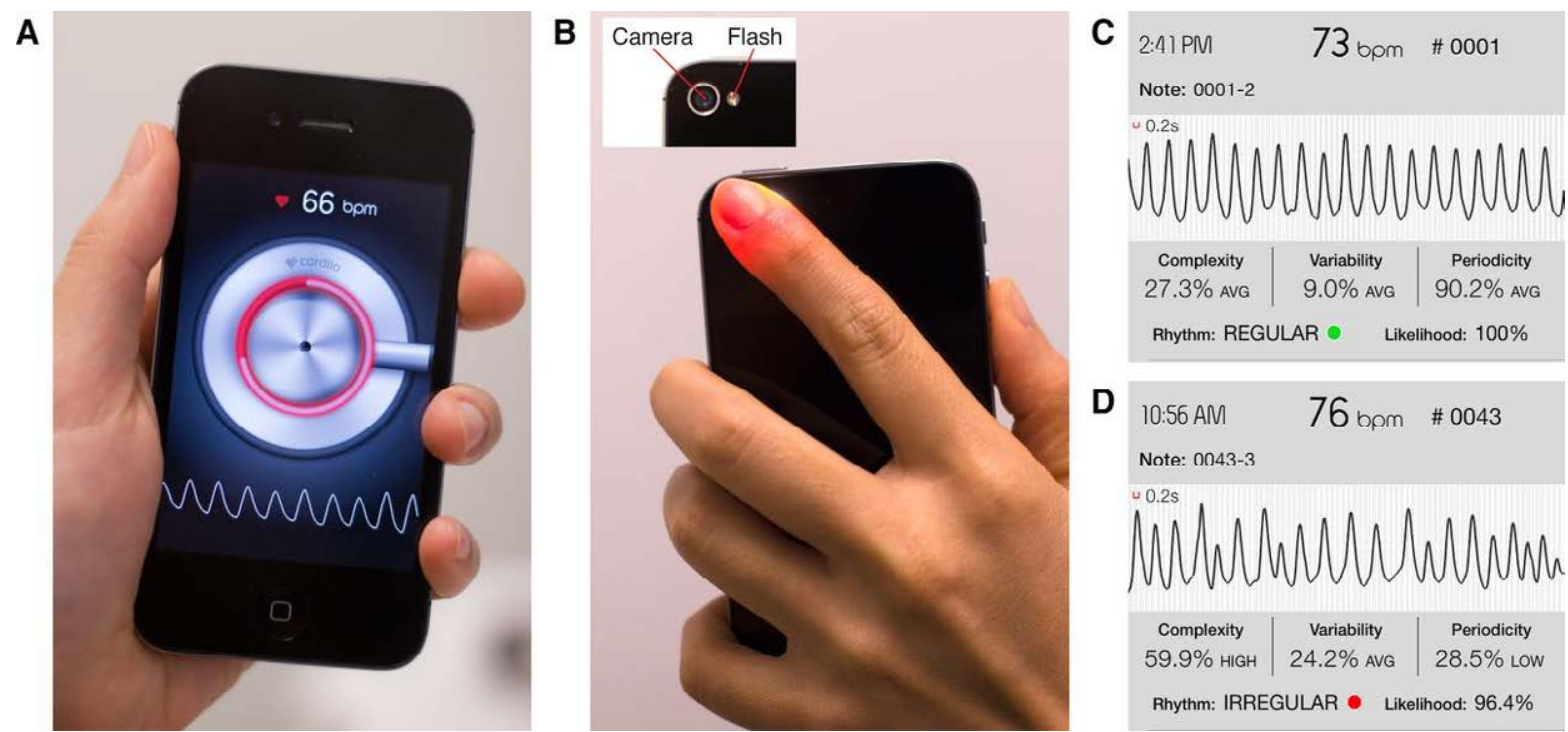

Figure 1. Cardio Rhythm Application

(A): Smartphone interface (B): Camera and Lamp (flash) required for waveform gathering (C): Example regular waveform output as captured by the device. (D): Example irregular waveform output as captured by the device.

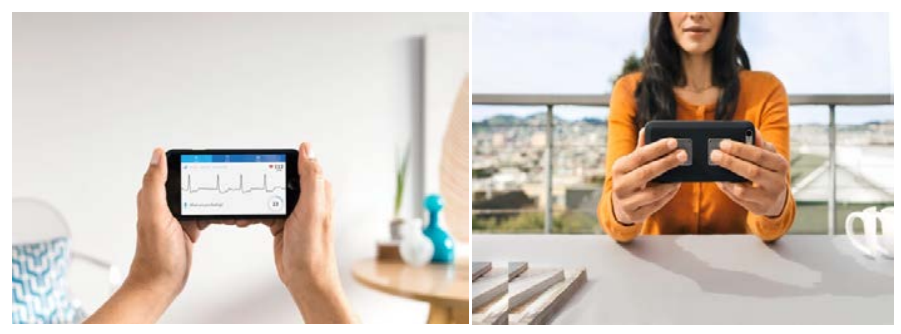

Figure 2. AliveCor Kardia Application (LEFT) and demonstration of finger placement for the AliveCor single-lead ECG (right).

sound signal, and transmitted to a smartphone on which the AliveCor Kardia App has been installed. The tracings can be reviewed on the smartphone, electronically stored, or electronically sent for review by the user's provider (Figure 2).

AliveCor has developed three FDA-cleared detectors or algorithms for use in the device [7]. These detectors approximate ECG Lead I, with the patient placing fingers from each hand on the respective electrodes. The rhythm is labeled as "normal" when the patient's heart rate is between 50-100 beats per minute, there are no or very few abnormal beats, and the shape, timing, and duration of each beat is considered normal. The rhythm is labeled as "unreadable" when the detector indicates there was too much interference for an adequate recording, whether from too much movement, or poor contact between the electrodes and the patient's skin. The rhythm is labeled as "Possible AF Detected" when the device detects the presence of AF, and has been shown to do so with $98 \%$ sensitivity and $97 \%$ specificity when comparing it with a contemporaneous 12-lead ECG interpreted by a cardiologist [8]. AliveCor notes that this device provides data and is not capable of making a diagnosis of AF. The recorded ECG can then be sent to a physician or medical professional for further review.

\section{Smartphone studies}

The accuracy of the AliveCor device has been investigated by multiple studies [8-10]. The sensitivity and specificity of the AliveCor device was assessed by Lau et al. [8]. They sought to assess and enhance the initial AF detection algorithm, as well as to assess the accuracy of the device as a tool for the detection of AF by comparing it with a simultaneous 12-lead ECG interpreted by a cardiologist. In order to assess the initial algorithm and further enhance it, 109 patients (39 with AF) were recruited. Following a 12-lead ECG, each patient had a single lead (Lead I) iPhone ECG which was later presented to two cardiologists blinded to the 12-lead diagnosis. The actual rhythm was determined by the 12-lead ECG interpreted by a third cardiologist. Following unbinding, the algorithm was optimized by increasing the weighting of absence of $\mathrm{P}$ waves, and applied to the same dataset. To validate the optimized rhythm, a total of 204 patients, including 48 in AF, were recruited. Data were collected in the same manner with blinding to the 12-lead diagnosis, and analyzed in the same way. They reported a high sensitivity (98\%), specificity (97\%), and accuracy (97\%) of the optimized AF detection algorithm to detect AF.

The cost-effectiveness of using the AliveCor device to screen for AF was investigated in the Screening Education and Recognition in Community pharmacies of Atrial Fibrillation to prevent stroke in an ambulant population aged $\geq 65$ years study (SEARCH-AF) by Lowres et al. [9]. Pharmacists performed pulse palpation and iECG recordings collected by the AliveCor device. In their investigation $(n=1000)$, the automated AF detection algorithm showed a sensitivity of $98.5 \%$ and a specificity of $91.4 \%$ when compared to an over-read performed by a cardiologist. Using treatment/outcome data from a United Kingdom cohort of 5,555 patients with incidentally detected asymptomatic AF, they determined the cost-effectiveness would be $\$ 4,066$ per Quality Adjusted Life Year gained and \$20,695 for preventing one stroke. The effectiveness of the AliveCor device in identifying AF was investigated by Williams et al. [10]. A total of 99 patients were recruited to the study. In their study, sensitivity was reported to be $90-93 \%$ and specificity was 76-86\% when compared to 12-lead ECG. The usability and accuracy of the AliveCor device for AF screening in a hospital population with an increased risk for AF was investigated by Desteghe et al. [11]. A total of 445 hospitalized patients in cardiology or geriatric wards were recruited for the study. A single-lead ECG captured by the AliveCor device was compared to a full 12-lead or 6-lead ECG recording. In this setting, they reported the device to have a sensitivity of $81.8 \%$ and a 
specificity of $94.2 \%$. Including device patients (pacemaker or ICD), in the analysis resulted in a sensitivity of $36.8 \%$ and a specificity of $96.1 \%$.

While these studies succeeded in establishing the sensitivity and specificity of the device, no study to date has yet to investigate the utility of a mobile health intervention in affecting clinical outcomes. The iPhone Helping Evaluate Atrial Fibrillation Rhythm through Technology study (iHEART) [12] is a single center, prospective, randomized controlled trial which seeks to accomplish this goal. In this study, a total of 300 participants with a recent history of atrial fibrillation will be enrolled. Participants will be randomized 1:1 to receive either the iHEART intervention, receiving an iPhone with an AliveCor Mobile ECG and behavioral altering motivational text messages, or usual cardiac care for 6 months. Outcomes assessed will include the difference in recurrent AF detection rate over the six-month study period between the control group and the iHEART intervention group, as well as the time-to-treatment for those treated for recurrent AF.

\section{Summary}

Application-based smartphone arrhythmia detectors provide a low barrier to use, as they require no additional hardware beyond a smartphone. However, there are still questions as to whether these applications are reliable. In fact, there are currently no validated PPG applications for the detection of arrhythmias available on the Apple App Store or the Google Play Store. The Cardio Rhythm application, mentioned above, is still in the developmental stages and not available for download to the general population. That being said, an initial validation study indicates that the Cardio Rhythm application, when made available, could provide an accurate and reliable means to detect $\mathrm{AF}$ in patients at risk of developing AF.

Smartphone accessory-based arrhythmia devices currently offer a validated means of monitoring atrial fibrillation. The AliveCor device mentioned above has received FDA approval and has undergone multiple studies to investigate its accuracy. In the United State, the device is available over the counter and is marketed directly to the general public with a manufacturer suggested retail price of $\$ 99.00$ (US). Multiple controlled studies have shown the device to be a reliable and accurate means of detecting atrial fibrillation. However, it is important to note that sensitivity is greatly decreased in patients with an implantable device and is not recommended for patients with pacemakers or ICDs. The detection of AF by the AliveCor device is not diagnostic, and positive findings of new AF should warrant a confirmatory ECG. The utility of this device will be further investigated by the iHEART study, which seeks to compare the AliveCor device to the current standard of care in a real-world setting. These devices show promise in arrhythmia assessment, managing patients with $\mathrm{AF}$, and diagnosing AF early in high risk patients. Caution should be used when assessing data provided by these devices, as validation in a real-world setting is still underway. That being said, these devices may be more reliable than symptom recognition, leading to quicker follow-up with a confirmatory ECG or other testing.

\section{References}

1. Smith A (2015) U.S. Smartphone Use in 2015. Pew Research Center.

2. Mozaffarian D, Benjamin EJ, Go AS, Arnett DK, Blaha MJ, et al. (2016) Executive Summary: Heart Disease and Stroke Statistics-2016 Update: A Report From the American Heart Association. Circulation 133: 447-454. [Crossref]

3. Wolf P A, Abbott RD, Kannel WB (1991) Atrial Fibrillation as an Independent Risk Factor for Stroke: the Framingham Study. Stroke 22: 983-988. [Crossref]

4. Healey JS, Connolly SJ, Gold MR, Israel CW, Van Gelder IC, et al. (2012) Subclinical atrial fibrillation and the risk of stroke. $N$ Engl J Med 366: 120-129. [Crossref]

5. Coyne KS, Paramore C, Grandy S, Mercader M, Reynolds M, et al. (2006) Assessing the direct costs of treating nonvalvular atrial fibrillation in the United States. Value Health 9: 348-356. [Crossref]

6. Chan PH, Wong CK, Poh YC, Pun L, Leung WW, et al. (2016) Diagnostic Performance of a Smartphone-Based Photoplethysmographic Application for Atrial Fibrillation Screening in a Primary Care Setting. J Am Heart Assoc 5: e003428. [Crossref]

7. AliveCore. AliveCor FAQ. https://www.alivecor.com/faq/. Retrieved January 2017.

8. Lau JK, Lowres N, Neubeck L, Brieger DB, Sy RW, et al. (2013) IPhone ECG application for community screening to detect silent atrial fibrillation: A novel technology to prevent stroke. Int J Cardiol 165: 193-194. [Crossref]

9. Lowres N, Neubeck L, Salkeld G, Krass I, McLachlan AJ, et al. (2014) Feasibility and cost-effectiveness of stroke prevention through community screening for atria fibrillation using iPhone ECG in pharmacies: The SEARCH-AF study. Thromb Haemost 111: 1167-1176. [Crossref]

10. Williams J, Pearce K, Benett I, Williams J, Manchester M, et al. (2015) The effectiveness of a mobile ECG device in identifying AF: sensitivity, specificity and predictive value. British Journal of Cardiology 22: 70-72.

11. Desteghe L, Raymaekers Z, Lutin M, Vijgen J, Dilling-Boer D, et al. (2016) Performance of handheld electrocardiogram devices to detect atrial fibrillation in a cardiology and geriatric ward setting. Europace 19: 29-39. [Crossref]

12. Hickey KT, Hauser NR, Valente LE, Riga TC, Frulla AP, et al. (2016) A single-center randomized, controlled trial investigating the efficacy of a mHealth ECG technology intervention to improve the detection of atrial fibrillation: the iHEART study protocol. BMC Cardiovasc Disord 16: 152. [Crossref]

Copyright: (C2017 White RD. This is an open-access article distributed under the terms of the Creative Commons Attribution License, which permits unrestricted use, distribution, and reproduction in any medium, provided the original author and source are credited. 